\title{
Intransitive Temporal Multi-Agent's Logic, Knowledge and Uncertainty, Plausibility
}

\author{
Vladimir Rybakov \\ School of Computing, Mathematics and DT, Manchester Metropolitan University, \\ Chester Street, Manchester M1 5GD, U.K. (full time) and Mathematical Institute, \\ Siberian Federal University, Krasnoyarsk, Russian Federation (part time) \\ V.Rybakov@mmu.ac.uk
}

\begin{abstract}
We study intransitive temporal logic implementing multiagent's approach and formalizing knowledge and uncertainty. An innovative point here is usage of non-transitive linear time and multi-valued models - the ones using separate valuations $V_{j}$ for agent's knowledge of facts and summarized (agreed) valuation together with rules for computation truth values for compound formulas. The basic mathematical problems we study here are - decidability and decidability w.r.t. admissible rules. First, we study general case - the logic with non-uniform intransitivity and solve its decidability problem. Also we consider a modification of this logic - temporal logic with uniform non-transitivity and solve problem of recognizing admissibility in this logic.
\end{abstract}

Keywords: temporal logic, multi-agent's logic, non-transitive time, deciding algorithms, knowledge, admissible rules.

\section{Introduction}

In the area of applications logic to Computer Science worthy place is occupied by temporal logic. It works very efficiently in various subdivisions of CS, Information Sciences and KR. An important version of temporal logic for CS, - LTL - linear temporal logic (with UNTIL and NEXT), was introduced by Z. Manna, and A. Pnueli in late 1980'. Since then, many impressive results concerning pure logical properties of LTL (e.g. decidability and axiomatization) were obtained (cf. e.g. Gabbay and Hodkinson[9-11], Vardi [26,27]). An essential component of information sciences is the notion of knowledge - a highly reliable information which is collected up to the moment and has some particular importance. 
The approach to concept of knowledge in CS via multi-agent environment, when the knowledge to be obtained via agent's discussions, cooperation, evaluations, computational experiments, etc. formed a solid branch in CS (cf. e.g. [29-31], [14,5]). An interpretation of knowledge in a multi-agent logic with distances was offered in Rybakov et al [22]), an algorithm solving satisfiability problem was found. Concept of Chance Discovery in multi-agent's environment was studied in Rybakov [23, 24]), a logic defining uncertainty via agents opinions was studied in McLean et al [15]). Representation of agent's interaction as a dual of the logical operation formalizing common knowledge (which earlier was suggested in Fagin et al [7]) was elaborated in Rybakov [21,20].

Approach to model knowledge in terms of symbolic logic appeared (probably first time) in Hintikka [13] in his book Knowledge and Belief. Now the field of knowledge representation and reasoning about knowledge in logical framework is very popular area. Various modal and multi-modal logics were used for modeling agents reasoning. In particular, multi-modal logics were used for this purpose in Balbiani et al [6], Vakarelov [28], Fagin et al [7], Rybakov [17, 20]. Modern study of knowledge and believes in terms of single-modal logic was undertaken in Halpern et al [12].

Concept of justification in terms of epistemic logic makes an another viewangle on knowledge (cf. e.g.. Artemov et al $[2,3])$. The problem of rational agents and its effect to logical omniscience problem is studied recently (cf. Artemov, et al [1]).

This our paper investigates intransitive temporal logic implementing multiagent's approach and formalizing knowledge and uncertainty in this framework. An innovative point here is usage of non-transitive linear time and multi-valued models - the ones using separate valuations $V_{j}$ for agent's knowledge of facts and summarized (agreed) valuation and rules for computation truth valued for compound formulas. We illustrate how the notion of knowledge and uncertainty might be represented in such framework. The basic mathematical problems we study here are the fundamental ones for any logical system - decidability and decidability w.r.t. admissible rules. First we consider very general case - the logic with non-uniform intransitivity and solve its decidability problem. The problem of recognizing admissible rules in this logic remains open. Next, we consider a modification of this logic - temporal logic with uniform non-transitivity and solve problem of recognizing admissibility in this logic.

\section{Notation, Logical Language, Brief Motivation}

To make our paper easy readable (without looking for external literature) we very briefly recall necessary definitions and notation. The language of Linear Temporal Logic (LTL in sequel) extends the language of Boolean logic by operations $\mathbf{N}$ (next) and $\mathbf{U}$ (until).

Formation rules for LTL-formulas built up from a set Prop of propositional letters are as follows: any letter of Prop is a formula. The set of all formulas is closed w.r.t. applications of Boolean operations, the unary operation $\mathbf{N}$ (next) 
and the binary operation $\mathbf{U}$ (until). Informal interpretation of the formula $\mathbf{N} \varphi$ is: $\varphi$ holds in the next time point (state). A formula $\varphi \mathbf{U} \psi$ has meaning: $\varphi$ will be true until $\psi$ first time will be true. Standard semantic models for LTL are the following infinite linear Kripke structures.

A model is a quadruple $\mathcal{M}:=\langle\mathcal{N}, \leq$, Next, $V\rangle$, where $\mathcal{N}$ is the set of all natural numbers; $\leq$ is the standard linear order on $\mathcal{N}$, Next is the binary relation, where $a$ Next $b$ is true iff $b$ is the number next to $a$, that is $b=a+1$.

The valuation $V$ for a set of letters $P \subseteq$ Prop is a mapping which assigns truth values to elements of $S$. That is, for any $p \in S, V(p) \subseteq \mathcal{N}$. The set $V(p)$ is the set of all $n$ from $\mathcal{N}$ where $p$ is true (w.r.t. $V$ ).

The triple $\langle\mathcal{N}, \leq$, Next $\rangle$ from the above is said to be a Kripke frame (which we will denote in sequel for short by $\mathcal{N}$ ). For any Kripke model $\mathcal{M}$, the truth values via $V$ for the propositional letters are extended to arbitrary formulas as follows:

$$
\begin{aligned}
& \forall p \in \operatorname{Prop}(\mathcal{M}, a) \Vdash_{V} p \Leftrightarrow a \in \mathcal{N} \wedge a \in V(p) ; \\
& (\mathcal{M}, a) \vdash_{V}(\varphi \wedge \psi) \Leftrightarrow(\mathcal{M}, a) \Vdash_{V} \varphi \wedge(\mathcal{M}, a) \vdash_{V} \psi ; \\
& (\mathcal{M}, a) \vdash_{V} \neg \Leftrightarrow \operatorname{not}\left[(\mathcal{M}, a) \Vdash_{V} \varphi\right] ; \\
& (\mathcal{M}, a) \Vdash_{V} \mathbf{N} \varphi \Leftrightarrow \forall b\left[(a \text { Next } b) \Rightarrow(\mathcal{M}, b) \vdash_{V} \varphi\right] ; \\
& (\mathcal{M}, a) \Vdash_{V}(\varphi \mathbf{U} \psi) \Leftrightarrow \exists b\left[(a \leq b) \wedge\left((\mathcal{M}, b) \Vdash_{V} \psi\right) \wedge\right. \\
& \left.\forall c\left[(a \leq c<b) \Rightarrow(\mathcal{M}, c) \Vdash_{V} \varphi\right]\right] .
\end{aligned}
$$

A formula $\varphi$ is said to be valid in the model $\mathcal{M}$ (denotation $-\mathcal{M} \Vdash \varphi$ ) if, for any $b$ from $\mathcal{M}(b \in \mathcal{N}),(\mathcal{M}, b) \Vdash_{V} \varphi$. The linear temporal logic LTL is the set of all formulas which are valid in all models.

The aim of our paper is to investigate linear logic with intransitive time. Therefore we briefly motivated our assumption about non-transitivity. Why we may assume that time might be non-transitive, what we mean by that? Here we consider time as a computational resource (e.g. its admitted length), as an individual human perception of time, as a background for collection and elicitation of knowledge.

Let us start from the individual perception of time in our human memory. We sense time as a sequence of events which we remember, we perceive it as a linear discrete succession (since we do not memorize very many events within few seconds). Our human memory if limited, finite. This means that what we knew and remembered a year ago might not be in our memory now; what we knew ten years ago may be not remembered by us a year ago. This says about intransitivity of human memory about time events in past.

Consider now computational aspect of time as a resource for analysis of results. Time events while computational runs may be recorded in protocols of computation for inspection and references. Any protocol is a finite sequence of records and not all necessary information might be found there. Though protocol may give references to other older protocols recorded in earlier computations. Here the time in applicational (not philosophical) aspect looks as non-transitive.

Assume that we work with extraction of data from databases. Data may be recorded in DBs and the storage of any one is finite. Any DB is recorded during 
a finite interval of time and may be incomplete. The procedure of updating DBs is a sequence of actions in time. This sequence may have terminating points, and what an old DB may contain could be already omitted in the updated one, so DBs knowledge in time is non-transitive.

Is we consider multi-agents reasoning than time events may be viewed as an individual ones (with effects as pointed above) and else this multi-agents brings its own effects. E.g. the amount of agents participating in taking decisions may be changed during some intervals of time; it might be not-uniform and to swell the same as to shrink. The priority of experts views may be changed, etc. And if experts view on truth of a statement was affirmative five years ago it may be opposite now. So, agents knowledge in time environment looks as intransitive.

\section{Temporal Multi-Agent's Modes, Temporal Logic}

Our approach is based on non-transitive temporal logic $\mathcal{L} \mathcal{T} \mathcal{L}_{N T}$ and technique allowing to find its decision algorithm (cf. Rybakov [25]). We start from giving precise definition of our new, modified models and description of rules for computation truth values of formulas. Then we first comment how these new models may represent multi-agent information, knowledge and uncertainty, give some illustrating examples.

Definition 1. An intransitive linear frame is a tuple

$$
\mathcal{F}:=\left\langle N, \leq, \text { Next }, \bigcup_{j \in N}\left[R_{j}\right]\right\rangle,
$$

components of which are as follows.

- $N=\bigcup_{i \in \operatorname{In\subset N}}\left[i, m_{i}\right]$ ([i, $\left.m_{i}\right]$ is the interval of all natural numbers situated between $i$ and $\left.m_{i}\right)$. The set $I n$ is a set of indexes - it is a subset of $N$;

- $\forall i_{1}, i_{2} \in I n, i_{1} \neq i_{2} \Rightarrow\left(i_{1}, m_{i_{1}}\right) \cap\left(i_{2}, m_{i_{2}}\right)=\emptyset$;

- $\forall i \in \operatorname{In}\left(m_{i}>i\right)$; for any $j \in\left[i, m_{i}\right]$ any $R_{j}$ is the standard linear order on the interval $\left[j, m_{i}\right]$;

- Next is the standard NEXT relation on $N$ : $\mathbf{n}$ Next $\mathbf{m}$ if $m=n+1$.

For the sequel we fix notation: $t(i):=m_{i}$ - boundary of transitivity for $i$. The multi-agent's models $\mathcal{M}$ on such frames $\mathcal{F}$ are defined by fixing valuations $V_{i}, i \in A,\|A\|<\infty$ for a set of letters $P$ - agents valuations for truth of letters $p \in P,-$, i.e. $\forall i, \forall p \in P, V_{i}(p) \subseteq N$.

$A$ is a set of indexes for agents, for each model it may be different (any model may have its own fixed agents, their quantity may be different). For all $n, n \in V_{i}(p)$ is interpreted as $p$ is true at the state $n$ by opinion of the agent $i$. Also we consider the agreed (global) valuation $V$ for letters from $P$ : -

$$
\mathbf{V}(\mathbf{p})=\left\{\mathbf{n} \mid \mathbf{n} \in \mathbf{N},\left\|\left\{\mathbf{i} \mid \mathbf{i} \in \mathbf{A}, \mathbf{n} \in \mathbf{V}_{\mathbf{i}}(\mathbf{p})\right\}\right\|>\mathbf{k}\right\},
$$

where $k$ is a fixed rational number (for this given model), which is bigger than $\|A\| / 2$. 
That is $k$ is the threshold, which shows that the number of the agents which are sure that $p$ is true in the given state (world) is big enough, bidder than half.

The particular value of $k$ may vary from model to model - each one has its own threshold. Now any such model $\mathcal{M}$ is a multi-valued model - with a finite number of different valuations.

The logical language for our logic based at such models is an extension of the one for LTL from previous section. We extend it by agent's knowledge operations $A_{i}, i \in A$ applied to only letters - for all $p \in P, A_{i}(p)$ is a formula. We introduce rules for computation truth on models $\mathcal{M}$ for formulas as follows. For letters and boolean operations it is standard: $\forall p \in P, \forall n \in N,(\mathcal{F}, n) \Vdash_{V} p \Leftrightarrow p \in$ $V(p) ;(\mathcal{F}, n) \Vdash_{V} \alpha \wedge \beta \Leftrightarrow\left[(\mathcal{F}, n) \Vdash_{V} \alpha\right.$ and $\left.(\mathcal{F}, n) \Vdash_{V} \beta\right]$; etc. For operation $\mathbf{N}$ next, it is standard again:

$$
\forall n \in N,(\mathcal{M}, n) \vdash_{V} \mathbf{N} \varphi \Leftrightarrow\left[(n N \text { ext } m) \Rightarrow(\mathcal{M}, m) \vdash_{V} \varphi\right] .
$$

But $\mathbf{U}$ - until operation, and agents operations work in a non-standard way, since the models are intransitive and since agents truth operations work as nominals. We suggest the following rules:

Definition 2. For any formulas $\varphi$ and $\psi$,

$$
\begin{gathered}
\forall n \in N,(\mathcal{M}, n) \Vdash_{V}(\varphi \mathbf{U} \psi) \Leftrightarrow \\
\exists m\left[\left(n R_{n} m\right) \wedge\left((\mathcal{M}, m) \Vdash_{V} \psi\right) \wedge \forall k\left[(n \leq k<m) \Rightarrow(\mathcal{M}, k) \Vdash_{V} \varphi\right]\right] ; \\
\forall n \in N, \forall i \in A,(\mathcal{M}, n) \Vdash_{V} A_{i}(p) \Leftrightarrow n \in V_{i}(p) .
\end{gathered}
$$

The agent's knowledge operations $A_{i}$, as we see, are applied to only letters but not to temporal compound formulas. The reason for it is as follows. The origin of the problem comes from our definition of models as multi-valued models (when each agent has own valuation of propositional letters; but we see that approach is very natural and well corresponding to multi-agents reasoning).

If we would allow the operations $A_{i}$ to be freely applied to arbitrary compound formulas and sub-formulas, then usage them in temporal (and modal) formulas would immediate cause clash/conflict in computation truth values.

E.g. if we have a formula $A_{i}(\varphi)$ with temporal formula $\varphi$, we either have to redefine truth values for letters $p$ always in now and future (which means to ignore knowledge of other agents), or to resolve what to do with all other possible agent's sub-formulas $A_{j}\left(p_{m}\right)$ w.r.t. the agent $i$. Thus, it might be that we need to give some agents a preference, but it is not clear what for to make an advantage to some ones. So, because this uncertainty, we prefer to let these cobwebs for future research and to study first this basic case.

It is easy to accept that this approach correspond well to our intuition about multi-agent information and time. The agents have own knowledge about facts, we code it by $A_{i}(p)$. But the rules for computation compound formulas are already objective, general and global, the same for all agents. Though, to consider 
different rules for computation truth values for compound, nested formulas looks as an attractive and promising idea.

The logic we wish to introduce is the collection of all general statements, formulas which are valid in all models.

Definition 3. The multi-agent non-transitive logic TMA Int is the set of all formulas which are valid in all models $\mathcal{M}$.

This logic is temporal, and therefore we may define via $\mathbf{U}$ the modal operations $\square$ and $\diamond$ in standard way: $\diamond \varphi:=\top \mathbf{U} \varphi, \square \varphi:=\neg \diamond \neg \varphi$. The logic is intransitive which allows such formulas as e.g.

$$
\square p \wedge \diamond \mathbf{N} \neg p
$$

to be satisfiable. Indeed - it is sufficient to take the model with all $m_{i}-i=3$ and $p$ to be true on the interval $[1,2,3]$ and to be false elsewhere.

The understanding (formal definition(s)) of knowledge, uncertainty and plausibility may be convincingly interpreted if we will consider the models with time and NEXT directed to past (not to future). We may easy agree that knowledge is coming from past, but not from the future.

The past time - in our human memory (or in storage of information in DBs from previous experience, length of protocols for completed computations, etc) evidently looks as non-transitive. Indeed, any database contains records stored in a finite amount of time, though it may contain information where to look for earlier events (so to say - to use NEXT - pointer to a new time interval).

We may understand knowledge as facts, statements which are convincingly true for all period of time which we remember (at least for leading part of experts, agents). This locally, in models, to be expressed by formula $\square \varphi$ : $\varphi$ was always true in past for dominating parts of experts (agents).

Then, the uncertainty (in this approach), may be interpreted as e.g. $\diamond \varphi \wedge$ $\diamond \neg \varphi$ - in some time points we remember agent's view for truth of $\varphi$ was supportive, and in some - the opinion of a majority was against. So, the truth for $\varphi$ in the interval of time which we remember was uncertain, not stable. Consider some more subtle example:

$$
\square[(\varphi \rightarrow[\diamond \neg \varphi \vee \mathbf{N} \neg \varphi]) \wedge(\neg \varphi \rightarrow[\diamond \varphi \vee \mathbf{N} \varphi])] .
$$

This formula expresses more delicate statement about uncertainty of truth values for $\varphi$ - it always oscillates.

Plausibility of $\varphi$ may be interpreted, e.g., as follows: $\neg \varphi \wedge \mathrm{N} \square \varphi$ : today experts hesitate about truth of $\varphi$, but always before today (admittedly - long time) they accepted it to be true. More example:

$$
\square \varphi \wedge \square \diamond \mathbf{N}^{2} \neg \varphi .
$$

This formula says that $\varphi$ is very plausible - as long as we remember with confidence, it was true, but in 2 steps above our reliable capacity of memory for past it was a state where it was false with some evidence. 
Many similar interpretations reflecting various subtleties of understanding uncertainty and plausibility may be suggested via this approach (e.g. using preference in opinion of most knowledgeable agents, and so forth).

\subsection{Technical part, decidability algorithm of $\mathbf{T M A}_{\text {Int }}$}

Now we turn to main technical problems solved in this paper, first we consider decidability of $\mathrm{TMA}_{\text {Int }}$. We will use here the approach from Rybakov [25] extending it for agent's knowledge operations.

An essential part of this approach is usage of the normal reduced forms for formulas, more exactly for inference rules to which formulas may be converted. It is very useful because it allows to avoid complicated calculations and evaluations for nested formulas. We will use reduction of formulas to inference rules (sequents). An inference rule is a sequent compound from the premises and the conclusion:

$$
\mathbf{r}:=\frac{\varphi_{1}\left(x_{1}, \ldots, x_{n}\right), \ldots, \varphi_{l}\left(x_{1}, \ldots, x_{n}\right)}{\psi\left(x_{1}, \ldots, x_{n}\right)} .
$$

Here $\varphi_{1}\left(x_{1}, \ldots, x_{n}\right), \ldots, \varphi_{l}\left(x_{1}, \ldots, x_{n}\right)$ and $\psi\left(x_{1}, \ldots, x_{n}\right)$ are formulas constructed out of letters (variables) $x_{1}, \ldots, x_{n}$. This rule formalizes simplest reasoning step: $\psi\left(x_{1}, \ldots, x_{n}\right)$ (which is called conclusion) follows (logically follows) from all formulas $\varphi_{1}\left(x_{1}, \ldots, x_{n}\right), \ldots, \varphi_{l}\left(x_{1}, \ldots, x_{n}\right)$.

Definition 4. We say that a rule $\mathbf{r}$ is valid in a model $\mathcal{M}$ if and only if the following holds:

$$
\left[\forall n\left((\mathcal{M}, n) \Vdash_{V} \bigwedge_{1 \leq i \leq l} \varphi_{i}\right)\right] \Rightarrow\left[\forall m\left((\mathcal{M}, m) \Vdash_{V} \psi\right)\right] .
$$

Otherwise we say $\mathbf{r}$ is refuted in $\mathcal{M}$, or refuted in $\mathcal{M}$ by $V$, and write $\mathcal{M} \nVdash_{V}$ $\mathbf{r}$. A rule $\mathbf{r}$ is valid in a frame $\mathcal{F}$ (notation $\mathcal{F} \Vdash \mathbf{r}$ ) if it is valid in any model based at $\mathcal{F}$.

Usage of inference rules for decidability problem (verification if a formula is a theorem for our logic) is based at the following simple fact. Given a formula $\varphi$, we transform $\varphi$ into the rule $x \rightarrow x / \varphi$. Then it is evident that

Lemma 1. Formula $\varphi$ is a theorem of TMA Int (that is $\varphi \in T M A_{\text {Int }}$ ) iff the rule $(x \rightarrow x / \varphi)$ is valid in any frame $\mathcal{F}$.

Thus, we bring decidability of formulas to decidability of rules; but surprisingly it simplifies the problem. We will use the rules in the reduced form.

Definition 5. We say that a rule $\mathbf{r}$ has reduced normal form if $\mathbf{r}=\varepsilon / x_{1}$ where

$$
\varepsilon:=\bigvee_{1 \leq j \leq l}\left[\bigwedge_{1 \leq i \leq n} x_{i}^{t(j, i, 0)} \wedge \bigwedge_{1 \leq i \leq n}\left(\mathbf{N} x_{i}\right)^{t(j, i, 1)} \wedge \bigwedge_{m \in A, 1 \leq i \leq n}\left(A_{m} x_{i}\right)^{t(j, m, i, 1)} \wedge\right.
$$




$$
\left.\bigwedge_{1 \leq i, k \leq n, i \neq k}\left(x_{i} \mathbf{U} x_{k}\right)^{t(j, i, k, 1)}\right]
$$

always $t(j, i, m), t(j, i, k, 1), t(j, m, i, 1) \in\{0,1\}$ and, for any formula $\alpha$ above, $\alpha^{0}:=\alpha, \alpha^{1}:=\neg \alpha$.

Definition 6. A rule $\mathbf{r}_{\mathbf{n f}}$ in reduced normal form is a normal reduced form for a given rule $\mathbf{r}$ iff, for any frame $\mathcal{F}$ for $T M A_{\text {Int }}, \mathcal{F} \Vdash \mathbf{r} \Leftrightarrow \mathcal{F} \Vdash \mathbf{r}_{\mathbf{n f}}$.

Theorem 1. For any given rule $\mathbf{r}$ we can construct in (single) exponential time some it's reduced normal form $\mathbf{r}_{\mathbf{n}}$.

Proof is rather simple and short. It is sufficient to specify the language of our logic to the general algorithm described in e.g. Lemma 5 from [4] and to follow closely its proof. We may consider the rules with only single premise, so let $r=\alpha / \beta$ be an inference rule. For $r, S u b(r)$ be the set of all subformulas of $r$. We need a set of new variables $Z=\left\{z_{\gamma} \mid \gamma \in \operatorname{Sub}(r)\right\}$.

Let us consider the rule in the intermediate form:

$$
r_{\mathrm{if}}=z_{\alpha} \wedge \bigwedge_{\gamma \in \operatorname{Sub}(r) \backslash \operatorname{Var}(r)}\left(z_{\gamma} \leftrightarrow \gamma^{\sharp}\right) / z_{\beta},
$$

where

$$
\gamma^{\sharp}= \begin{cases}z_{\delta} * z_{\epsilon} & \text { when } \gamma=\delta * \epsilon \text { for } * \in\{\wedge, \vee, \rightarrow, \mathbf{U}\} . \\ * z_{\delta} & \text { when } \gamma=* \delta \text { for } * \in\left\{\neg, \mathbf{N}, A_{m}, m \in A\right\},\end{cases}
$$

The rules $r$ and $r_{\text {if }}$ are equivalent w.r.t. truth at any model. Indeed, suppose $M$, be a model with a valuation $V$ over its frame such that $M \|_{V} r$. Then $M \Vdash_{V} \alpha$ and there exists an element $w \in N$, such that $(M, w) \forall_{V} \beta$. Let $W$ be the valuation defined as follows: $W\left(z_{\gamma}\right)=V(\gamma)$. It is straightforward to show that $M \Vdash_{W} z_{\alpha} \wedge \bigwedge_{\gamma \in \operatorname{Sub}(r) \backslash \operatorname{Var}(r)}\left(z_{\gamma} \leftrightarrow \gamma^{\sharp}\right)$. In addition, $(M, w) \forall_{W} z_{\beta}$.

For the other direction, suppose $M \Vdash_{W} z_{\alpha} \wedge \bigwedge\left\{z_{\gamma} \leftrightarrow \gamma^{\sharp} \mid \gamma \in \operatorname{Sub}(r) \backslash \operatorname{Var}(r)\right\}$ and $(M, w) \forall_{W} z_{\beta}$, for some valuation $W: Z \rightarrow 2^{N}$ and some $w \in N$. Define $V: \operatorname{Var}(r) \rightarrow 2^{N}$ by $V\left(x_{i}\right)=W\left(z_{x_{i}}\right)$. It follows directly that for all $\gamma \in \operatorname{Sub}(r)$, $V(\gamma)=W\left(z_{\gamma}\right)$. Thus $M \Vdash_{V} \alpha,(M, w) \forall_{V} \beta$, hence $M \forall_{V} r$.

Finally, we transform the premise of the obtained rule $r_{\text {if }}$ into a perfect disjunctive normal form over primitives of the form $x_{i}, \mathbf{N} x_{i}, A_{m} x_{i}$ and $x_{i} \mathbf{U} x_{j}$. This requires no more than exponential time on the number of variables, i.e., on the number of sub-formulas of the original rule (the same as for reduction of any boolean formula to the perfect disjunctive normal form). Q.E.D.

Based at this reduction of formulas to rules in reduced forms and technique borrowed from Rybakov [25] we may prove

Theorem 2. The satisfiability problem for $T M A_{\text {Int }}$ is decidable. There is an algorithm which, for any given formula, verifies its satisfiability, and computes a valuation satisfying it in a special finite model $\mathcal{F}(N(r)$ ) if it is satisfiable (at next stage we can transform this model in a standard infinite model). 
Here we extend the proof from [25] to adopt usage of agents knowledge operations $A_{m}$ (following closely to the original proof). Thus, the logic TMA Int $_{\text {is decidable; }}$ this is first main technical result of our paper.

\section{Problem of Admissibility}

Far the more complicated decidability problem is decidability w.r.t. admissible inference rules. We would like to study admissibility problem for a logics from suggested background. Recall that a rule

$$
\mathbf{r}:=\frac{\varphi_{1}\left(x_{1}, \ldots, x_{n}\right), \ldots, \varphi_{l}\left(x_{1}, \ldots, x_{n}\right)}{\psi\left(x_{1}, \ldots, x_{n}\right)},
$$

is said to be admissible in a logic $L$ if, for every tuple of formulas, $\alpha_{1}, \ldots, \alpha_{n}$, we have $\psi\left(\alpha_{1}, \ldots, \alpha_{n}\right) \in L$ whenever $\forall i\left[\varphi_{i}\left(\alpha_{1}, \ldots, \alpha_{n}\right) \in L\right]$.

The solution of the admissibility problem for the logic LTL itself (i.e. finding an algorithm recognizing admissibility of inference rules) was obtained in Rybakov, 2008, [19] (cf. also [18]), basis for rules admissible in LTL was found in Babenyshev and Rybakov, 2011, [4].

We have to specify the notion of admissibility for inference rules in our multiagent's logics because we use agent's knowledge operations $A_{i}$ which cannot be used above nested formulas.

Definition 7. A given rule

$$
\mathbf{r}:=\frac{\varphi_{1}\left(x_{1}, \ldots, x_{n}\right), \ldots, \varphi_{l}\left(x_{1}, \ldots, x_{n}\right)}{\psi\left(x_{1}, \ldots, x_{n}\right)},
$$

is said to be admissible in the logic TMA Int if, for every tuple of formulas, $\alpha_{1}, \ldots, \alpha_{n}$, we have $\psi\left(\alpha_{1}, \ldots, \alpha_{n}\right) \in T M A_{\text {Int }}$ whenever $\forall i\left[\varphi_{i}\left(\alpha_{1}, \ldots, \alpha_{n}\right) \in\right.$ $\left.T M A_{\text {Int }}\right]$, where for any $x_{i}$ above if $x_{i}$ has at least one occurrence in $r$ in form $A_{j}\left(x_{i}\right)$ then $\alpha_{i}=x_{i}$.

The restriction for substitutions above is necessary since our multi-agent logic cannot admit nested formulas bounded by agent's knowledge operations $A_{j}$. A restriction for substitutions in defining admissibility was already considered in literature (cf. for instance, - Odintsov, Rybakov - [16]). We currently cannot answer the question about recognizing admissibility in the logic $\mathrm{TMA}_{\text {Int }}$ from previous section, but we are able to do it for its restricted version - the one for models with bounded intransitivity.

Definition 8. A temporal frame $\mathcal{F}$ with uniform non-transitivity $m$ is a particular case of frames for $T M A_{\text {Int }}$

$$
\mathcal{F}:=\left\langle N, \leq, \text { Next }, \bigcup_{i \in N}\left[R_{i}\right]\right\rangle
$$

given in Definition 1 in Section 3, when any interval $[i, t(i)]$, has length $m$, where $m$ is a fixed natural number (measure of intransitivity). 
So, the only distinction from our general case in the previous section is that instead of arbitrary measure on intransitivity $m_{i}$ for any world $i$, we consider the same and fixed one - $m$. It looks as we assume that models (objective world), not agents, always must remember the same interval of the time in past - the one with length $m$. Then we define models on such frames as we did earlier above (bearing in mind the presence multi-agent's valuations for agent's knowledge about truth the facts and agreed truth valuation $V$ ).

Definition 9. The logic TMA Int,m is the set of all formulas which are valid at any model $\mathcal{M}$ with the measure of intransitivity $m$.

The definition of admissibility for inference rules in this logic is exactly the same as we defined above in this section for $\mathrm{TMA}_{\text {Int }}$. It seems that to consider and to discuss such logic is reasonable, since we may put limitations on the size of time intervals that agents (experts) may introspect in future (or to remember in past). An easy observation concerning the logic $\mathrm{TMA}_{\text {Int }, m}$ itself is that it is decidable: it is trivial (since for verification if a formula of temporal degree $k$ is a theorem of $\mathrm{TMA}_{\text {Int }, m}$ we will need to check it on only initial part of the frames consisting only $k+1$ subsequent intervals of length $m$ ). One more immediate observation is:

Proposition 1. TMA Int $m \nsubseteq T M A_{\text {Int }}$ for all $m$.

Proof is evident since

$$
\left(\bigwedge_{i \leq m}\left[p \wedge \mathbf{N}^{i} p\right] \rightarrow \square p\right) \in \mathrm{TMA}_{I n t, m}
$$

The main technical result of this section is solution of the admissibility problem for logics $\mathrm{TMA}_{\text {Int }, m}$.

Theorem 3. For any $m$, the linear temporal logic with uniform non-transitivity TMA $A_{\text {Int }, m}$ is decidable w.r.t. admissibility of inference rules.

The proof is essentially other than the one for decidability w.r.t. admissible rules of the linear (transitive) temporal logic LTL itself (given in [19]). Presence of infinite sequence intransitivity intervals in the models makes the case different.

\section{Open problems}

We think the following open questions could be of interest:

(i) Decidability of $\mathrm{TMA}_{\text {Int }}$ itself w.r.t. admissible inference rules.

(ii) Decidability w.r.t. admissible rules of the variant of TMA $\mathrm{Tnt}_{\text {, } m}$ with nonuniform intransitivity, when intransitivity intervals are of length at most $m$, but the length may be different.

(iii) The problems of axiomatization for $\mathrm{TMA}_{\text {Int }}$ and $\mathrm{TMA}_{\text {Int }, m}$ are open. 


\section{References}

1. Artemov, S., Kuznets, R.: Logical Omniscience as Infeasibility. Ann. of Pure and Appl. Logic. 165, 6 - 25 (2014)

2. Artemov, S.: Justified Common Knowledge. Theoretical Computer Science. $357,4-22(2006)$

3. Artemov. S., Nogina, E.: Introducing Justification into Epistemic Logic . Journal of Logic and Computation. 15, 1059 - 1073 (2005)

4. Babenyshev. S., Rybakov, V.: Linear Temporal Logic LTL, Basis for Admissible Rules. Journal of Logic and Computation. 21, 1057 - 177 (2011).

5. Belardinelli, F., Lomuscio, A.: Interactions between Knowledge and Time in a First-Order Logic for Multi-Agent Systems: Completeness Results. Journal of Artificial Intelligence Research. 45, 1 - 45 (2012)

6. Balbiani, Ph., Vakarelov, D.: A Modal Logic for Indiscernibility and Complementarity in Information Systems, Fundam. Inform. 50, $243-263$ (2002)

7. Fagin, R., Halpern, J., Moses, Y., Vardi, M.: Reasoning about Knowledge, MIT Press (1995)

8. Friedman, H.: One Hundred and two Problems in Mathematical Logic. Journal of Symbolic Logic. 40, 113 - 130 (1975)

9. Gabbay, D.M., Hodkinson, I.M., Reynolds, M.A.: Temporal Logic. Mathematical Foundations and Computational Aspects. Clarendon Press, Oxford (1994)

10. Gabbay, D.M., Hodkinson, I.M.: An Axiomatization of the Temporal Logic with Until and Since over the Real Numbers. Journal of Logic and Computation. 1, 229 - 260 (1990).

11. Gabbay, D., Hodkinson, I.: Temporal Logic in Context of Databases. In: Copeland, J. (editor) Logic and Reality, Essays on the legacy of Arthur Prior, Oxford University Press (1995)

12. Halpern, J., Samet, D., Segev, E.: Defining Knowledge in Terms of Belief. The Modal Logic Perspective. Review of Symbolic Logic. 2, 469 - 487 (2009).

13. Hintikka, J.: Knowledge and Belief: An Introduction to the Logic of the Two Notions. Cornell University Press, Ithaca, (1962)

14. Lomuscio, A., Michaliszyn, J.: An Epistemic Halpern-Shoham Logic. In: Proceedings of the 23rd International Joint Conference on Artificial Intelligence (IJCAI13), AAAI Press, Beijing, China, 2013, pp. 1010 - 1016 (2013)

15. McLean, D., Rybakov, V.: Multi-Agent Temporary Logic $T S 4_{K_{n}}^{U}$ Based at Non-linear Time and Imitating Uncertainty via Agents Interaction. In: Artificial Intelligence and Soft Computing, LNCS, vol. 7895, pp. 375 - 384, Springer, Heidelberg (2013).

16. Odintsov, S., Rybakov, V.: Inference Rules in Nelson's Logics, Admissibility and Weak Admissibility. Logica Universalis. 9, 93 - 120 (2015)

17. Rybakov, V.V.: Refined Common Knowledge Logics or Logics of Common Information. Archive for Mathematical Logic, 42, 179 - 200 (2003)

18. Rybakov, V.V.: Logical Consecutions in Discrete Linear Temporal Logic. J. of Symbolic Logic. 70, 1137 - 1149 (2005)

19. Rybakov, V. V.: Linear Temporal Logic with Until and Next, Logical Consecutions. Annals of Pure and Applied Logic. 155, 32 - 45 (2008)

20. Rybakov, V.: Logic of Knowledge and Discovery via Interacting Agents Decision Algorithm for True and Satisfiable Statements. Information Sciences. $179,1608-1614$ (2009) 
21. Rybakov, V.: Linear Temporal Logic $L T L_{K_{n}}$ extended by Multi-Agent Logic $K_{n}$ with Interacting Agents. Journal of logic and Computation. 19, 989-1017 (2009)

22. Rybakov, V., Babenyshev, S.: Multi-agent Logic with Distances based on Linear Temporal Frames. In: Artificial Intelligence and Soft Computing, LNCS, vol. 6114, pp. 337 - 344. Springer, Hidelberg (2010)

23. Rybakov, V.: Chance Discovery and Unification in Linear Modal Logic. - In: Knowledge-Based and Intelligent Information and Engineering Systems (KES 2011), LNCS, vol. 6882 pp. 478 - 485. Springer, Heidelberg (2011)

24. Rybakov, V.V.: Logical Analysis for Chance Discovery in Multi-Agents' Environment. In: Frontiers in Artificial Intelligence and Applications, vol. 243, pp. 1593 - 1601. Springer, Heidelberg (2012)

25. Rybakov, V.: Non-Transitive Linear Temporal Logic and Logical Knowledge Operations. J. of Logic Computation, Oxford Press, doi: 10.1093/logcom/exv016 (2015)

26. Vardi, M.: An Automata-theoretic Approach to Linear Temporal Logic. In: Y.Banff Higher Order Workshop, pp. 238 - 266, Available at http://citeseer.ist.psu.edu/vardi96automatatheoretic.htm (1995)

27. Vardi, M. Y.: Reasoning about the Past with Two-way Automata. In: Larsen., K.G., Skyum., S., Winskel., G. (eds) ICALP, LNCS, vol. 1443, pp. 628 - 641, Springer, Heidelberg (1998)

28. Vakarelov, D.: A Modal Characterization of Indiscernibility and Similarity Relations in Pawlak's Information Systems. In: Rough Sets, Fuzzy Sets, Data Mining and Granular Computing, LNCS, vol. 3641, pp. 12 - 22. Springer, Heidelberd (2005)

29. Wooldridge, M., Lomuscio, A.: Multi-Agent VSK Logic.. In: Proceedings of the Seventh European Workshop on Logics in Artificial Intelligence (JELIAI2000), Springer-Verlag (2000)

30. Wooldridge, M.: An Automata-theoretic Approach to Multi-agent Planning. In: Proceedings of the First European Workshop on Multi-agent Systems (EUMAS 2003), Oxford University (2003)

31. Wooldridge, M., Huget, M., Fisher, M., Parsons, S.: Model Checking MultiAgent Systems: The MABLE Language and its Applications. International Journal on Artificial Intelligence Tools, 15, 195 - 225, (2006) 